\title{
Local RF-Heating by Using High-Density Nanostructured Magnetic Garnet Ceramic
}

\author{
M Bañobre-López', T Gaudisson ${ }^{2}, J_{\text {Rivas }}^{3}$, S Ammar ${ }^{2}$ and R Valenzuela ${ }^{4}$ \\ ${ }^{1}$ International Iberian Nanotechnology Laboratory, Braga, Portugal \\ 2ITODYS, Université de Paris-Diderot, Sorbonne Paris Cité, CNRS, France \\ ${ }^{3}$ Department of Applied Physics, Universidade de Santiago de Compostela, Spain \\ ${ }^{4}$ Instituto de Investigaciones en Materiales, Universidad Nacional Autónoma de México, México
}

\begin{abstract}
A localized RF-heating system can be built in an arrangement similar to typical hyperthermia measurements, just by substituting the nanoparticle suspension by a consolidated ferro- or ferrimagnetic material. In this work, we show that such an arrangement allows the controlled RF-heating of small bodies (typically for annealing or curing of integrated circuits). Yttrium iron garnet, $\mathrm{Y}_{3} \mathrm{Fe}_{5} \mathrm{O}_{12}(\mathrm{YIG}$ ), prepared by a novel technique involving a soft chemistry synthesis followed by consolidation by Reactive Spark Plasma Sintering, was chosen as ferrimagnetic material. A study of the resulting heating phenomenon is presented, and its general features are discussed. It appears that in the case investigated, the main heating process is associated with the magnetic hysteresis of the material.
\end{abstract}

Keywords

RF-heating, Nanostructured YIG, Magnetic hysteresis, Coercive field

\section{Introduction}

Magnetic hyperthermia of nanoparticle (NP) suspensions has been among the most attractive research subjects in the last 20 years, and impressive progress and developments have been made, especially in the perspective of antitumor applications [1,2]. Radiofrequency (RF) energy has also been applied directly (i.e., no NPs involved) in many medical devices to induce a variety of metabolic processes in tissues [3]. To the best of our knowledge, however, RF technical heating by means of a consolidated magnetic material subjected to an AC magnetic field has not been studied so far.

RF local heating by means of materials with proper geometry can be very useful in a variety of applications, such as annealing integrated circuits on flexible plastic sub- strates [4] or curing processes in wafers [5]. The heating rate and the stabilization temperature can be controlled in a straightforward way by playing on the amplitude and frequency of the AC magnetic field. The size and geometry of the magnetic material could allow a finely localized heating, preserving areas sensitive to high temperatures.

In this letter, we present a basic study of the phenomena in order to associate the main performance of the technique to the magnetic properties of a given magnetic material. Measurements similar to hyperthermia techniques were carried out on platelets of yttrium iron garnet. Yttrium iron garnet, $\mathrm{Y}_{3} \mathrm{Fe}_{5} \mathrm{O}_{12}$ ("YIG") was chosen because it is a soft ferrimagnetic material, with a saturation magnetization (in the bulk state) of about 26.8 $\mathrm{Am}^{2} / \mathrm{kg}$ at room temperature, and a Curie temperature of $560 \mathrm{~K}$; in the undoped state, it possesses a high elec-

*Corresponding author: $R$ Valenzuela, Instituto de Investigaciones en Materiales, Universidad Nacional Autónoma de México, P.O. Box 70-360, Coyoacán, Ciudad de México, 04510, México, E-mail:monjaras@unam.mx

Received: April 25, 2018: Accepted: July 04, 2018: Published: July 06, 2018

Copyright: (c) 2018 Bañobre-López M, et al. This is an open-access article distributed under the terms of the Creative Commons Attribution License, which permits unrestricted use, distribution, and reproduction in any medium, provided the original author and source are credited. 
trical resistivity of at least $10^{12} \Omega \mathrm{cm}$ [6-8]. YIG is virtually the material with the lowest electromagnetic losses; it has the smallest linewidth in ferromagnetic resonance (FMR) [9]. YIG is a kind of model material, often chosen for basic studies on magnetization processes [10]. For this work, the samples were synthesized and consolidated by a combination of two novel techniques; first the synthesis of precursors by hydrolysis in a polyol [11], and then their consolidation by the reactive spark plasma sintering (RSPS) process [12]. This method leads to high density, fine grain microstructures by sintering processes at lower temperatures and shorter times than the classic solid-state sintering [13].

\section{Materials and Methods}

The sample preparation started with a polyol synthesis [11] to produce the precursors. In order to obtain $10 \mathrm{~g}$ of $\mathrm{Y}_{3} \mathrm{Fe}_{5} \mathrm{O}_{12}, 18.5776 \mathrm{~g}$ of yttrium acetate $\mathrm{Y}\left(\mathrm{CH}_{3} \mathrm{CO}_{2}^{-}\right)_{3} \cdot 4 \mathrm{H}_{2} \mathrm{O}$ and $15.9301 \mathrm{~g}$ of iron acetate $\mathrm{Fe}\left(\mathrm{CH}_{3} \mathrm{CO}_{2}\right)_{2}$ were dissolved in diethylene glycol and brought to the boiling point of the solution $\left(\sim 200^{\circ} \mathrm{C}\right)$, at a heating rate of $6^{\circ} \mathrm{C} / \mathrm{min}$ under mechanical stirring and maintained in reflux and stirring for $3 \mathrm{~h}$. After cooling down to room temperature, the precipitated solid phase was recovered by centrifugation, washed with ethanol and dried overnight at $50^{\circ} \mathrm{C}$. After a pre-annealing in air at $400{ }^{\circ} \mathrm{C}$ for $2 \mathrm{~h}$, the powdered solid was processed by Reactive Spark Plasma Sintering (RSPS) [12] at $750^{\circ} \mathrm{C}$ in a graphite die for $15 \mathrm{~min}$, under vacuum and a uniaxial pressure of $100 \mathrm{MPa}$, to obtain a high-density pellet. Small platelets, typically a parallelepiped shape of a few $\mathrm{mm}$ by side, a fraction of $\mathrm{mm}$ thick, and tens of $\mathrm{mg}$ of mass, were cut from the pellets.

$\mathrm{X}$-ray diffraction studies (carried out with a Panalytical XperPro equipped with a X'celerator multichannel detector, using Co Ka radiation ( $\gamma=1.7889 \AA$ ) of the consolidated samples confirmed that a single garnet phase, corresponding to YIG unit cell was obtained. Scanning electron microscopy (SEM) (performed by means of a
Supra40 ZEISS FEG-SEM microscope operating at 5.0 $\mathrm{kV}$ ) was used to investigate the final microstructure of the consolidated samples.

Magnetization measurements of sintered YIG samples were carried out at $300 \mathrm{~K}$ in a LDJ 9600 VSM magnetometer (LDJ Electronics) with a maximum applied field of $10 \mathrm{kOe}$. In this technique, the sample is vibrated between two pick-up coils and their response is analyzed by means of a lock-in amplifier [14] to provide a measurement of the magnetization. The magnetic field is applied starting at the maximum field value $\left(+H_{\max }\right)$, slowly decreased and inverted to the negative value of the field $\left(-H_{\max }\right)$, and increased again to the initial value, thus describing a complete cycle. The total time to describe the full cycle is typically of a few minutes, so this result is often known as a "quasi static" hysteresis loop. The accuracy in both the magnetization measurements and the applied magnetic field determination in our system is better than $1 \%$ [15].

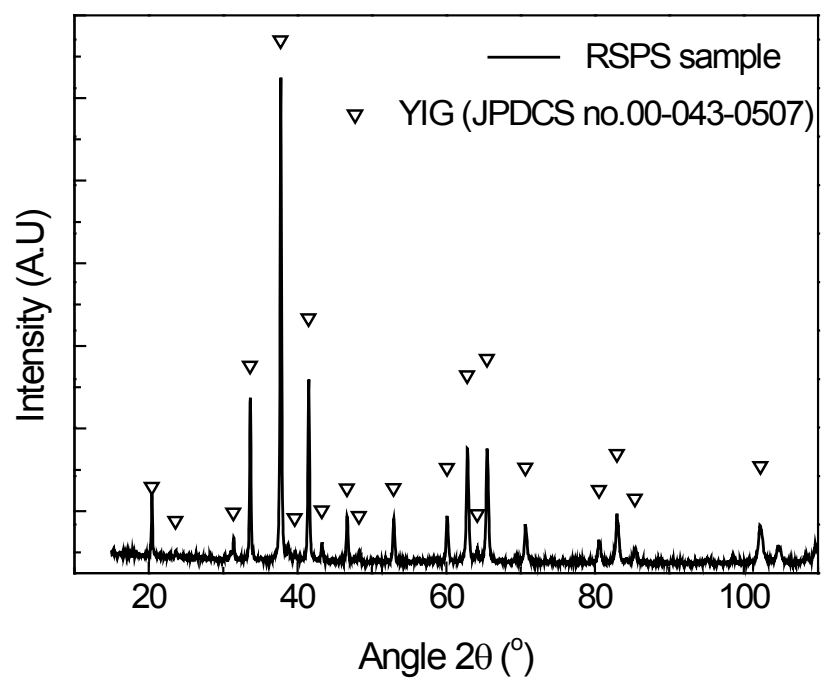

Figure 1: X-ray diffraction pattern of sintered samples (continuous line). The reported place of diffracted peaks (Card JPDCS No. 00-043-0507) is indicated by a triangle.
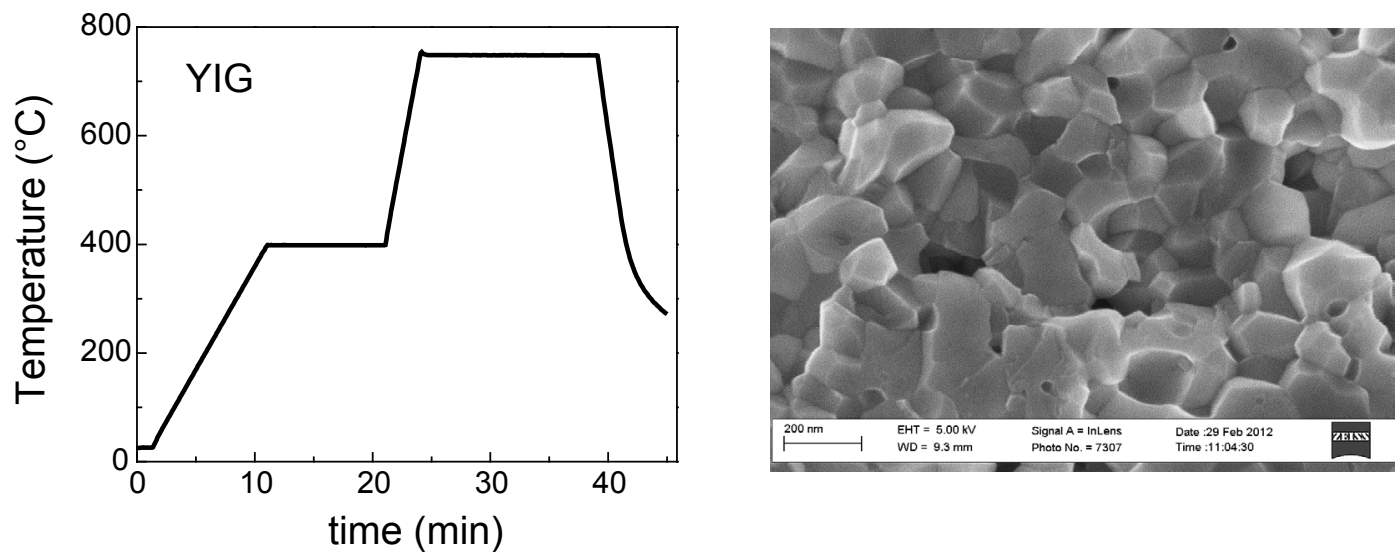

Figure 2: The temperature profile for the RSPS process is shown in the left; a $400^{\circ} \mathrm{C}$ plateau was applied to allow a degassing of the sample. At right, a micrograph of the obtained sample exhibited a grain size in the $150 \mathrm{~nm}$ range. 
Hyperthermia measurements were carried out in a nanoscale Biomagnetics system allowing to apply magnetic fields with amplitudes in the 0-300 Oe range at frequencies in the $250-869 \mathrm{kHz}$ range. Instead of using a suspension (as in typical hyperthermia measurements), the ceramic YIG platelet was directly fixed on the temperature sensing probe.

\section{Results and Discussion}

The crystal structure of the sintered samples showed a cubic symmetry in excellent agreement with the reported structure of yttrium iron garnet (JPDCS X-ray card number 00-043-0507), as shown in Figure 1. All the diffracted peaks corresponded to the YIG unit cell, so it can be concluded that a single-phase material was obtained. The RSPS process produced a nanostructured solid with high density and grains in the $150 \mathrm{~nm}$ range. The temperature profile of this RSPS process, as well as a micrograph of the resulting microstructure appear in Figure 2.

Measurements of the hyperthermia response of YIG

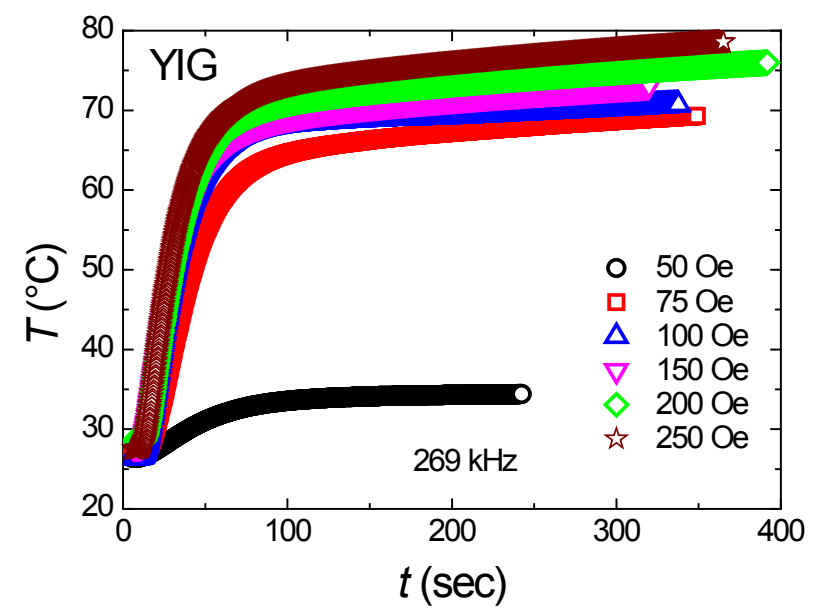

Figure 3: Hyperthermia results obtained on the YIG platelet at $269 \mathrm{kHz}$ and several amplitudes of the applied field in the 50-250 Oe range. platelets showed the expected increase in temperature as a function of time, as shown in Figure 3. The stabilization temperature increased as the applied field amplitude increased. However, a marked difference was observed for the smallest field amplitude (50 Oe), which exhibited a very limited increase in temperature as compared with the other applied fields.

These results exhibited a clear boundary in field amplitude between two magnetization processes. To analyze these results, the energy-absorbing processes should be considered. In hyperthermia phenomena, two main processes are taken into account: Néel relaxation, associated with the reorientation of the magnetization vector within free nanoparticles, and Brown relaxation, which occurs when the whole particle is reoriented, with magnetization remaining in an easy anisotropic direction. In the present case, the sample is a polycrystalline aggregate with grains tightly bound to each other, and the Brown relaxation can be ruled out. Therefore, the process depends only on the corresponding hysteresis loop.

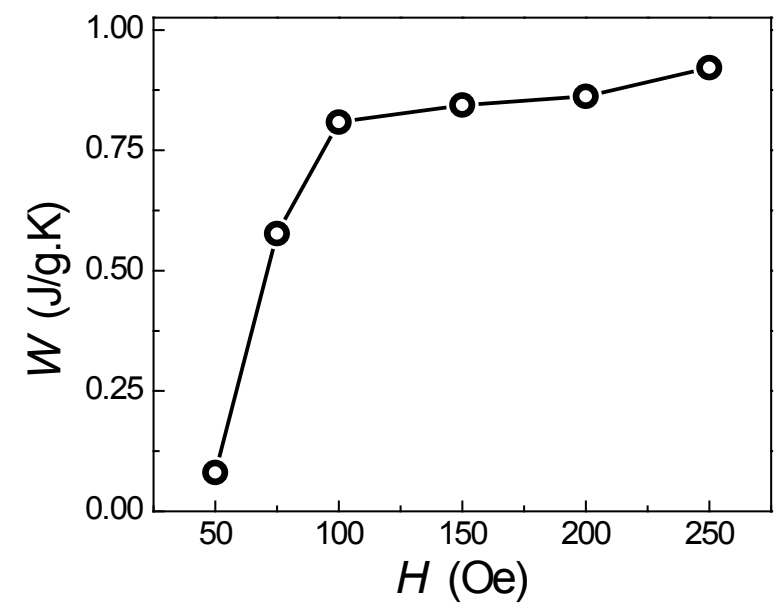

Figure 5: Total loss power (per unit mass and unit time), $\mathrm{W}$, as a function of the amplitude of the applied field, at a frequency of $269 \mathrm{kHz}$.
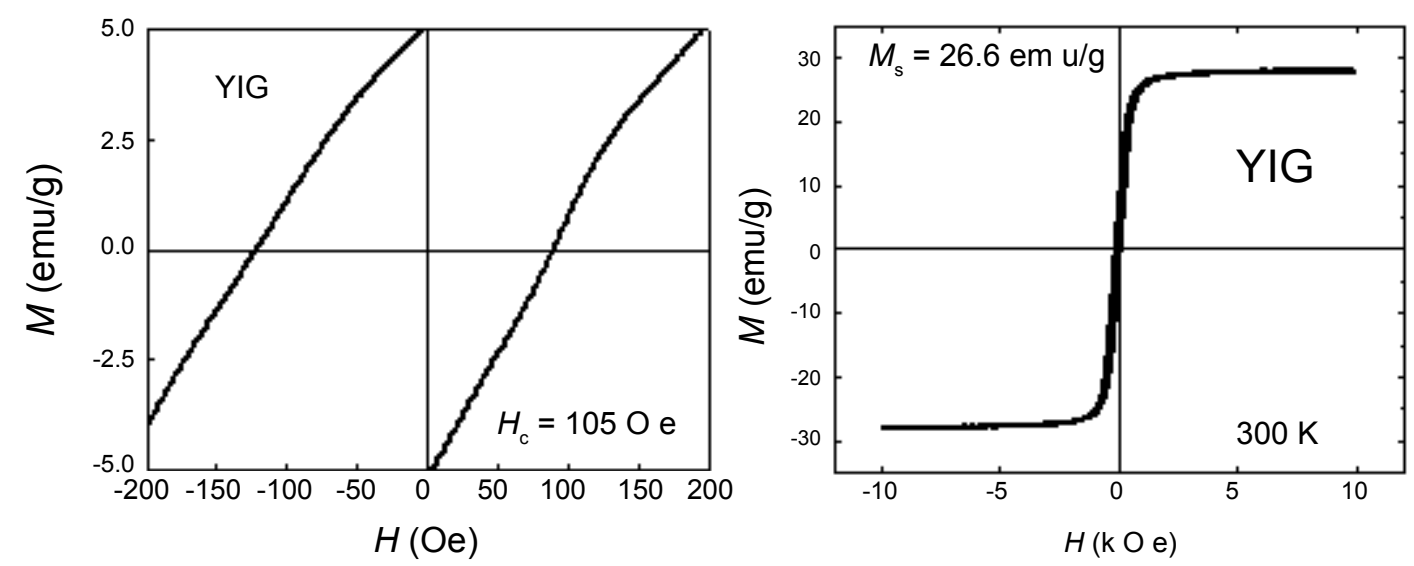

Figure 4: Hysteresis loop of the YIG platelet at room temperature. The applied field was oriented parallel to the platelet plane. The low field section is presented on the left, to show the coercive field (105 Oe). 
An examination of the hysteresis loops of this material showed that the limiting field is associated with the coercive field, $\mathrm{Hc}$ (105 Oe), which can be observed in Figure 4. For fields below $H c$, the $M=f(H)$ plot exhibits only minor hysteresis loops (not shown here), which involve a significantly smaller energy area, and hence, a smaller energy absorption.

The heat power, $Q$, involved in the process can be determined as:

$$
Q=m C p \Delta T
$$

Where $m$ is the magnetic material mass, $C p$ its specific heat and $\Delta T$ the rise in temperature. The total heat power, $W$ (loss power per unit mass and unit time), generated by the magnetic material can be expressed as [16]:

$$
\mathrm{W}=\mathrm{Q} / \Delta t m
$$

Where $\Delta t$ is the time interval. $W$ can then be evaluated from experimental values $\Delta T / \Delta t$ measured as the initial slope in $T$ vs. $t$ plots (Figure 3 ), as

$$
W=C \mathrm{p} \Delta T / \Delta t
$$

With the specific heat value for YIG [17], $C p=0.60$ $\mathrm{J} / \mathrm{g}$ K. The results are shown in Figure 5.

A plot of rise in temperature as a function of the field amplitude was made, see Figure 6, showing also a strong change in slope for fields below 100 Oe. Two different regimes appear, whose relations can be calculated, $T=$ $C_{1} H^{1.65}$ for $H<100$ Oe, and $T=C_{2} H^{0.10}$ for $H>100$ Oe, where $C_{1}$ and $C_{2}$ are constants. The first relationship could be compared with the Steinmetz equation [18], where the hysteresis loss (per volume and cycle) is customarily plotted against the peak value of the flux density as $W \sim B_{\max }{ }^{b}$. In the present case, it is the applied field amplitude, $H$, which is considered (instead of the peak

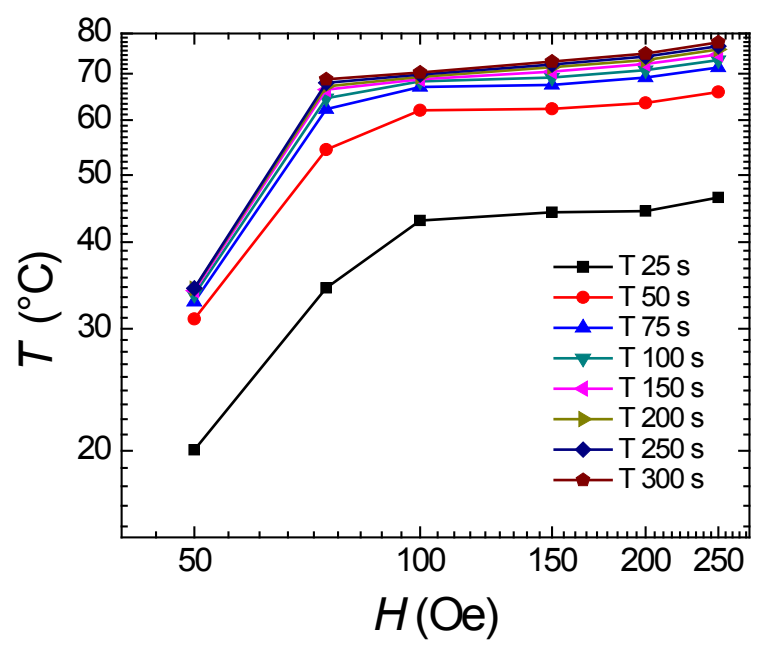

Figure 6: Relationship between rise in $T$ and field, $H$, for fixed times. Two slopes are observed, $T=C_{1} H^{1.65}$ for $H<$ $100 \mathrm{Oe}$, and $T=C_{2} H^{0.10}$ for $H>100 \mathrm{Oe}$. value of induction, $B$ ), as it is typically the significant parameter in hyperthermia.

The electrical resistivity in garnets is very high [6-8] and Eddy-current losses are not expected. Heating can therefore be associated mainly with magnetic hysteresis losses. For the high-field zone, $H>H_{c}$, the increase in temperature is high, but variations as a function of applied field amplitude are small, leading to a small exponent $(0.10)$. This feature can be associated with the fact that for this zone, flux variations are also quite small, as the material approaches magnetic saturation.

\section{Conclusions}

We presented a system allowing localized, tightly controlled thermal treatments on small samples, driven by AC magnetic fields. This system appears as especially suitable for curing and annealing integrated circuits. The most favorable magnetic properties of the heating element are those of a soft ferrimagnet: A reduced coercive field and a high saturation magnetization. These properties would allow an effective heating with a moderate field amplitude. For the material used, a garnet, which is an insulator, the power involved can be well explained by assuming losses associated only with magnetic hysteresis.

\section{References}

1. H Shokrollahi (2017) A review of the magnetic properties, synthesis methods and applications of maghemite. Journal of Magnetism and Magnetic Materials 426: 74-81.

2. AM Tishin, AA Shtil, AP Pyatakovm, VI Zverev (2016) Developing antitumor magnetic hyperthermia: Principles, materials and devices. Recent Patents on Anti-Cancer Drug Discovery 11: 360-375.

3. P Ihnat, LI Rudinska, P Zonca (2014) Radiofrequency energy in surgery: State of the art. Surgery Today 44: 985-991.

4. Q Cao, H Kim, M Pimparkar, JP Kulkarni, C Wang, et al. (2008) Medium-scale carbon nanotube thin-film integrated circuits on flexible plastic substrates. Nature 484: 495-500.

5. F Niklaus, RJ Kumar, JJ McMahon, J Yu, J-Q Lu, et al. (2006) Adhesive wafer bonding using partially cured benzocyclobutene for three-dimensional integration. Journal of the Electrochemical Society 153: 291-295.

6. DTT Nguyet, NP Duong, T Satoh, LN Anh, TD Hien (2012) Temperature-dependent magnetic properties of yttrium iron garnet nanoparticles prepared by citrate sol-gel. Journal of Alloys and Compounds 541: 18-22.

7. W Zhang, C Guo, R Ji, C Fang, Y Zeng (2011) Low-temperature synthesis and microstructure-property study of single-phase yttrium iron garnet (YIG) nanocrystals via a rapid chemical coprecipitation. Materials Chemistry and Physics 125: 646-651.

8. HP Fu, RY Hong, YJ Wu, GQ Di, B Xu, et al. (2008) Preparation and Faraday rotation of Bi-YIG/PMMA nanocomposite. Journal of Magnetism and Magnetic Materials 320: 2584-2590. 
9. N Mo, JJ Green, BA Beitscher, CE Patton (2007) High precision-metrology based microwave effective linewidth measurement technique. Rev Sci Instrum 78: 113903.

10. M Guyot, A Globus (1977) Determination of the domain wall energy and the exchange constant from hysteresis in ferromagnetic polycrystals. Journal de Physique (Paris).

11. S Ammar, A Helfen, N Jouini, F Fiévet, I Rosenman, et al. (2001) Magnetic properties of ultrafine cobalt ferrite particles synthesized by hydrolysis in a polyol medium. J Mater Chem 11: 186-192.

12. G Franceschin, N Flores-Martínez, G Vázquez-Victorio, S Ammar, R Valenzuela (2018) Sintering and reactive sintering by spark plasma sintering. In: Igor Shishkovsky, Sintering of functional materials. Tech Open Access Publisher, 124-145.

13. T Gaudisson, U Acevedo, S Nowak, N Yaacoub, J-M Greneche, et al. (2013) Combining soft chemistry and spark plasma sintering to produce highly dense and finely grained soft ferrimagnetic $\mathrm{Y}_{3} \mathrm{Fe}_{5} \mathrm{O}_{12}$ (YIG) ceramics. Journal of the American Ceramic Society 96: 3094-3099.

14. S Foner (1959) Versatile and sensitive vibrating-sample magnetometer. The Review of Scientific Instruments 30: 548-557.

15. LDJ Model 9600 Vibrating Sample Magnetometer Operation Manual. 3.

16. E Kita, H Yanagihara, S Hashimoto, K Yamada, T Oda, et al. (2008) Hysteresis power-loss heating of ferromagnetic nanoparticles designed for magnetic thermoablation. IEEE Transactions on Magnetics 44: 4452-4455.

17. SR Boona, JP Heremans (2014) Magnon thermal mean free path in yttrium iron garnet. Physical Review B 90: 064421.

18. P Parthasaradhy, SV Ranganayakulu (2014) Hysteresis and eddy current losses of magnetic material by Epstein frame method - novel approach. The International Journal of Engineering and Science 85-93. 